\title{
Utilizing arbitrary waveform generators to produce noise with imposed spectral characteristics
}

\author{
L. Angrisani, M. D’Apuzzo, M. D’Arco, E. Napoli, A. Strollo \\ Dipartimento di Ingegneria Elettrica e Tecnologie dell'Informazione, Università di Napoli Federico II, via Claudio 21, 80125 Napoli, Italy
}

ABSTRACT

The reliability and performance of many systems mostly depend of their noise rejection capability. The behavior of systems that are prone to noise is therefore investigated since the early production stage, as well as routinely during periodical maintenance actions. Actually, there are very few noise sources available on the market, the majority of which is capable of generating only noise characterized by a uniform power spectral density in a limited bandwidth; generators capable of producing colored noise are instead very rare. In theory, arbitrary waveform generators could be deployed and suitably programmed to serve as noise sources. But, the technicians charged of test and measurement assignments will barely find application notes that support them in stepping from theory to practice.

This work presents tutorial notes related to the simulation of colored noise, and proposes an analog generator that exploits an arbitrary waveform generator as noise source. The proposed generator is capable of producing noise signals characterized by arbitrary power spectral densities.

\section{Section: RESEARCH PAPER}

Keywords: Colored noise, filter design, digital-to-analog conversion, spectral spurs, Welch periodogram.

Citation: L. Angrisani, M. D’Apuzzo, M. D'Arco, E. Napoli, A. Strollo, Utilizing arbitrary waveform generators to produce noise with imposed spectral characteristics, Acta IMEKO, vol. 4, no. 1, article 4, February 2015, identifier: IMEKO-ACTA-04 (2015)-01-04

Editor: Paolo Carbone, University of Perugia

Received November $12^{\text {th }}, 2013$; In final form April 14 ${ }^{\text {th }}, 2014$; Published February 2015

Copyright: (C) 2014 IMEKO. This is an open-access article distributed under the terms of the Creative Commons Attribution 3.0 License, which permits unrestricted use, distribution, and reproduction in any medium, provided the original author and source are credited

Funding: This work was supported by the Science and Technology Division of the University of Naples 'Federico II' through the program FARO 2012.

Corresponding author: Mauro D’Arco, e-mail: darco@unina.it

\section{INTRODUCTION}

Noise has been extensively studied during the twentieth century. The first model that has been proposed to describe its randomness is the white noise model, which is, at present, widely used for analysing the behaviour of electronic systems. Sources that produce analogue white noise are also available on the market, and dedicated instruments that analyse the effects of the noise on the behaviour of complete electronic systems, but also single devices and components, are currently realized and marketed [1]-[3].

Noise sources are regularly employed in applications such as: the measurement of the frequency response of linear systems, the measurement of noise figures, the functional test of microwave and optoelectronic links. For instance, very fast full frequency response measurements can be gained using thermal noise, which is characterized by a flat spectrum that agilely spans whichever band of interest. The use of noise sources in conjunction with a calibrated instrument allows the measurement of noise figures of delicate equipment, such as mixers or receiver front ends. Radar and digital communication links are intermittently verified by means of built-in noise sources that are activated during off-times [4]; alternatively, the noise can be superimposed to signals including modulated data to perform measurements of the bit error rate (BER) curves also during on-times. The performance of optoelectronic systems that transmit data through fiber cables is also tested using noise sources in conjunction with phase modulators and oscillators to produce jitter in test signals [5]-[6]. For advanced encryption applications, robust random occurrences of digital codes can be produced by sampling a voltage generated by a real noise source: these codes require much more efforts to be cracked by hackers 
with respect to the pseudorandom codes produced by calculus schemes [7]. Finally, noise sources play an interesting role also in the enhancement of analogue-todigital converters performance [8] and in data encryption applications [9]. In fact, noise permits to enhance the linearity and the dynamic range of high-speed digital-toanalogue converters by means of dithering, which consists in adjoining noise characterized by spectral contents residing outside the band of interest [10]-[12].

In order to straightforwardly characterize noise and its effects common scalar noise metrics such as noise factor $(N F)$, equivalent noise temperature $\left(T_{e}\right)$, noise power $\left(P_{n}\right)$, $Y_{\text {Fact }}$ factor, and excess noise ratio $(E N R)$ are utilized in research and industrial applications. All these metrics are not independent form each other; for instance the equivalent noise temperature $T_{e}$ is a function of the noise factor $N F$, as well as the excess noise ratio combines the $Y_{F a c t}$ factor and noise factor $N F$. Besides the aforementioned metrics, an exhaustive characterization of noise can be gained by measuring the power spectral density (PSD). The PSD of any random process, such as noise, is defined as the Fourier transform of its autocorrelation function, anyway in practice, it is usually gained through more straightforward approaches. In particular, it is measured by means of spectrum analysers or FFT-based instruments, which provide the Fourier transform of a portion of the noise.

It is worth noting that many natural phenomena as well as many mechanisms that take place in modern systems produce noise that exhibits some different characteristics with respect to white noise, which is commonly referred to as coloured noise [13].

For many mechanisms that produce coloured noise, and especially for the most recently discovered ones, practical descriptive models are unavailable.

Also, the classical measurement approaches to characterize noise features are criticized. The calculation of scalar metrics such as the mean square value of the coloured noise could be not realistic as it happens in the presence of flicker or random walk noise [14]. Nonetheless, FFT-based approaches can be adopted only in the presence of white noise whereas produce biased results when applied to coloured noise. Actually, the use of FFT Analysers to measure noise spectral features is critical because they suffer from spectral leakage, which can be controlled only in the presence of repetitive signals, i.e. signals characterized by a discrete number of sinusoidal components. In these cases, coherent sampling or, alternatively, windowing can be used to counteract spectral leakage and obtain correct estimations of the magnitude of each component. Specifically, to get rid of spectral leakage, coherent sampling requires the acquisition and processing of a record of points including an exact number of cycles of the input signal; windowing instead provides the necessary tolerance for accurately measuring the magnitude of spectral components by reducing the broadband leakage at the expense of the narrowband one. Unfortunately, in the presence of noise, there are no means to counteract spectral leakage, because neither coherent sampling condition exists, nor windowing would be effective: any trade-offs between broadband and narrowband spectral leakage would not compensate bias effects. At the state of art, the most advanced solution to evaluate the spectral features of noise signals relies on the use of Welch's averaged, modified periodogram method. The Welch method is an FFT-based approach that involves averaging the results attained for partially overlapped segments of the input signal; averaging should mitigate bias effects and improve measurement accuracy and repeatability.

All the aforementioned issues related to applications, metrics, models and measurement techniques make plain why the use of coloured noise appears in theoretical approaches whereas, due to the superior complexity of a coloured noise generator, it is not common in experimental tests [15]-[16].

The paper shows how synthesizing digital coloured noise and, successively, how generating it into analogue form by exploiting the potentialities of arbitrary waveform generators [17]. The reader is thus provided with practical guidelines for the design of a programmable noise generator, which allows the user to choose the PSD of the noise he wants to produce. The proposed approach can be utilized anytime the equipment under test has to be stimulated by a signal with power residing in assigned frequency intervals and characterized by an arbitrary power spectral density. The main applications consist of: noise sensitivity analysis, characterization of sensors and actuators, and identification of linear and time invariant systems.

\section{NOISE WITH IMPOSED CHARACTERISTICS}

Different techniques to synthesize discrete-time coloured noise sequences have been proposed in the recent past. The proposed one shares with other existing techniques the basic approach of deriving coloured noise according to a general and well-known technique, namely, by filtering white noise with a linear and time invariant filter. The underlying theory is well known: the power spectral density of the output noise is shaped by the frequency response of the adopted filter. For input noise characterized by a uniform power spectral density, the PSD of the output noise coincides with the squared modulus of the filter frequency response. Therefore, to obtain a discrete random signal $y(n)$ with a pre-assigned real power spectral density $S_{y y}(v)$, the input white noise $w(n)$ is chosen as to have a unitary power density $S_{w w}(v)$, and the frequency response $H(v)=|H(v)| \mathrm{e}^{\mathrm{j} \beta(\gamma)}$ of the adopted filter as to satisfy:

$|H(v)|=\sqrt{S_{y y}(v)}$

Specifically, the proposed generator relies on the use of a filter with finite-impulse response. The user describes the shape of the frequency response of the filter, $H(v)$, through a frequency sampling approach. In other terms, the user designs the filter specifying the number of coefficients of the filter, the bandwidth $B$ in which he wants to confine the 
noise, and two short arrays of equal length, namely, a frequency array, freq, and a magnitude array, mag. The frequency array contains in ascending order frequency values within $(0,1)$ that are normalized values with respect to the bandwidth $B$. The magnitude array expresses the gain of the filter at the normalized frequency values given in freq. The short arrays typically contain a small number of elements, much less than the number of coefficients that the user may require to match precision goals. They serve to describe in a schematic way the desired PSD. The shape of the frequency response of the filter is then calculated through linear interpolation between the gain values defined in the magnitude array for a number of normalized frequencies equal to one half of the selected filter length. The phase response $\beta(v)$ of the filter cannot be arbitrarily chosen but has to satisfy the constraints of physical realization. As a rule, a linear phase response that introduces a suitable amount of delay can assure the physical realization. Among the different possibilities the delay can conveniently be chosen to avoid the use of complex arithmetic for the synthesis of the noise.

The procedure to synthesize the discrete-time noise with an imposed power spectral density can be definitively summarized as:

- $\quad$ selection of the number of coefficients, $2 L$, of the shaping filter;

- choice of the bandwidth $B$ that the PSD of the noise has to occupy;

- definition of the frequency and magnitude arrays, freq and mag, that specify the PSD of the target noise within the chosen bandwidth;

- calculation through interpolation of the $L$-sequence of the values, $\{|H(0)|,|H(1 / L)|,|H(2 / L)|, \ldots$ $|H(L / 2 L)|\}$, i.e. the values for the normalized frequency values, $v$, within the interval $(0,1 / 2)$;

- multiplication by -1 of the samples of the aforementioned $L$-sequence corresponding to odd normalized frequencies, $|H(1 / L)|,|H(3 / L)|$, $|H(5 / L)|, \ldots$; this operation confers to the filter the linear phase response:

$$
-j \beta(v)=-j \pi 2 L v
$$

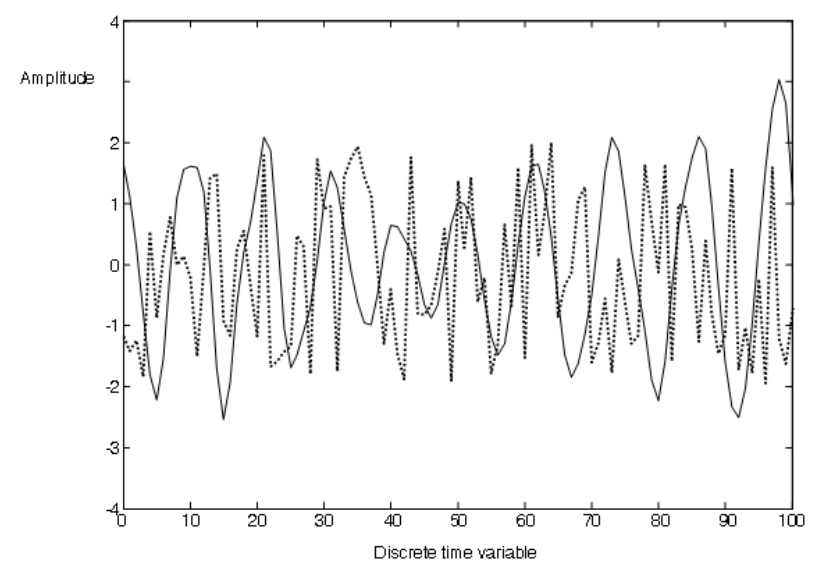

Figure 1. Graphics of the white noise (dotted line) and coloured noise (solid line) obtained by means of the shaping filter.

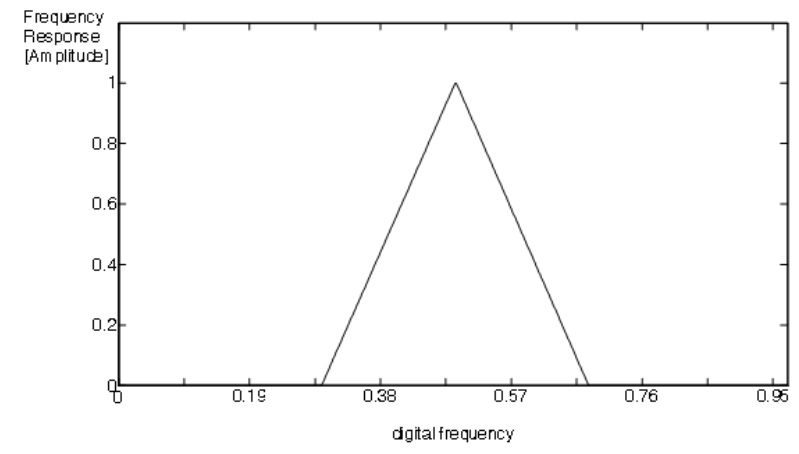

Figure 2. Frequency response in amplitude of the filter adopted to achieve coloured noise by filtering white noise. The filter is defined by the short digital frequency array freq $=\left[\begin{array}{lllll}0 & 0.30 & 0.50 & 0.70 & 1\end{array}\right]$ and magnitude array $\mathbf{m a g}=\left[\begin{array}{lllll}0 & 0 & 1 & 0 & 0\end{array}\right]$; the length of the filter is $2 L=512$. The filter assigns a triangular shape to the power spectral density of the noise; the power of the noise should be zero in the digital frequency intervals $(0,0.30)$ and $(0.70,1)$ and reaches a maximum value at 0.50 .

- specification of $H(v)$ in terms of the $2 L$ sequence $\{H(0), H(1 / L), H(2 / L), \ldots H(2 L 1 / 2 L)\}$ which gives the frequency response for normalized frequency values $v$ within the interval $(0,1)$. To this end the available $L$ sequence is prolonged through mirroring, which preserves the required Hermitian symmetry of the frequency response: samples at normalized frequencies $(L+i) / 2 L$ must have the complex conjugate values of those at normalized frequencies $(L-i) / 2 L, i$ ranging from 1 to $L-1$.

- calculation of the impulse response of the filter, $h(n)$, through the inverse discrete Fourier transformation of the aforementioned $2 L$-sequence.

As commentary, it is worth noting that the proposed filter is characterized by a frequency response expressed by coefficients which are all real numbers: no complex values are processed. The filter, being a finite impulse response filter, is inherently stable, thus the output never diverges. The causality of the filter is granted at the mere expense of an output delay: the first output sample is available after $L$ input samples. The sample rate related to the obtained noise signal is exactly twice its bandwidth.

As an example, Figure 1 shows portions of both a white noise and a coloured noise obtained filtering the former by

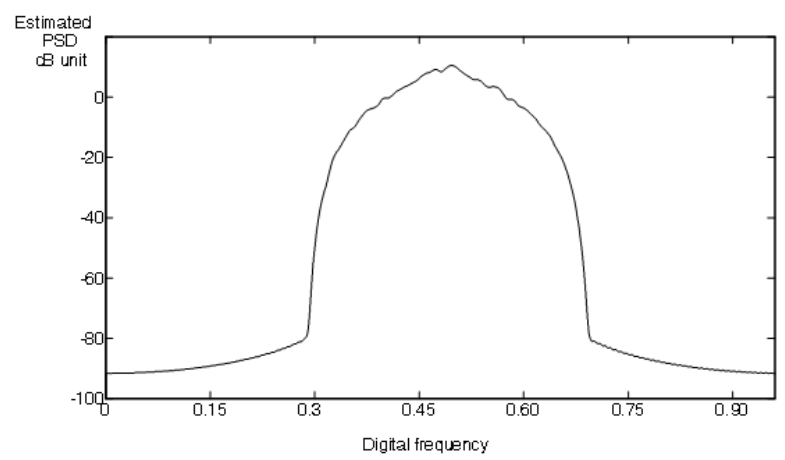

Figure 3. Power spectral density of the discrete noise estimated by means of the Welch periodogram. The logarithmic scale makes the triangular function appear as a bell-shaped curve. The residual noise in the digital frequency intervals $(0,0.30)$ and $(0.70,1)$, in which it is expected to be negligible, is at least $90 \mathrm{~dB}$ below the maximum power density level at $10 \mathrm{~dB}$. 
means of a shaping filter. The designed filter has the frequency response in amplitude given in Figure 2. It is defined by the short digital frequency array freq $=\left[\begin{array}{ll}0 & 0.3\end{array}\right.$ $0.5 \quad 0.7$ 1 $]$ and magnitude array mag $=\left[\begin{array}{lllll}0 & 0 & 1 & 0 & 0\end{array}\right]$, and gives to the PSD of the noise a triangular shape, characterized by symmetrical transition bands in the intervals $(0.3,0.5)$ and $(0.5,0.7)$, and stop bands in the intervals $(0,0.3)$ and $(0.7,1)$. Figure 3 shows the PSD of the discrete noise on a logarithmic scale as estimated by means of the Welch periodogram. Note that the logarithmic scale makes the triangular PSD appear as a bell-shaped curve. The power level of the residual noise in the digital frequency intervals $(0,0.3)$ and $(0.7,1)$, in which it is expected to be negligible, is at least $90 \mathrm{~dB}$ below the maximum power density level, which is equal to $10 \mathrm{~dB}$, when the length of the filter is $2 L=512$ taps.

The design of the filter could be optimized by windowing the obtained impulse response with an opportune window. There are many types of window such as: Bartlett, Blackman, Chebyshev, Gaussian, Hamming, Hanning, that could be thought of. Anyway, it is not convenient to perform optimization at this level, because when the discrete sequence is played in order to produce the analogue noise signal, the features of the synthesized noise are unavoidably modified, as it is illustrated in the next Section.

\section{GENERATING NOISE INTO ANALOGUE FORM}

In order to realize a programmable noise generator, i.e. a noise generator that allows the user to specify the PSD of the output noise, an arbitrary waveform generator can be exploited. As well known, arbitrary generators read from a waveform memory a stream of digital data that is given in input to a digital-to-analogue converter (DAC). The DAC operates as a clocked zero-order-hold circuit, hence the analogue noise signal is expected to be as a piece-constant waveform (in other terms the generator keeps constant any voltage level for a time interval equal to the duration of the clock period, and at the occurrence of the clock pulse performs a step change of the output voltage) [18]-[19].

The piece-constant nature of the analogue signal should not be jeopardized since it involves some undesired effect. In particular, aliases of the spectral contents of the analogue output are expected to appear in the frequency intervals adjacent to the band of interest and centred at multiple values of twice the selected bandwidth $B$.

To counteract the undesired effects of aliasing, which are inherent to the use of a zero-order hold circuit, the use of smoothing analogue filters is regularly deployed. All arbitrary waveform generators are equipped with one or, in the case of more expensive models, a few internal low-pass filters, which can be selected according to the particular waveform to be played. When the noise is filtered the step changes in the output voltage are smoothed and exhibit a finite rise time. The transient that characterizes the voltage change depends both on the type and the parameters of the filter.

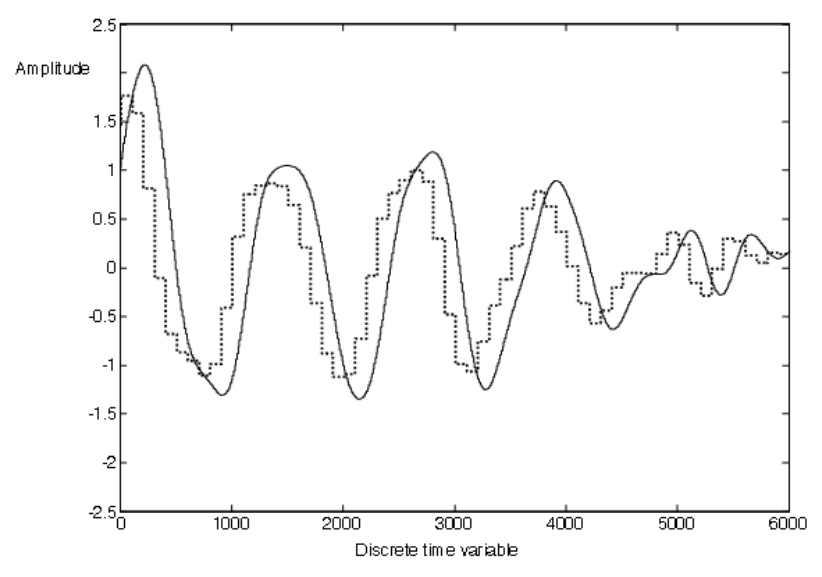

Figure 4. Digital noise signal as it should appear when played at full bandwidth by an arbitrary waveform generator (dotted line) and upsampled and interpolated version (solid line); an up-sampling factor equal to 10 has been utilized. Simulations have been performed utilizing 100 points for each voltage level, thus the dotted line is a piecewise line characterized by 100 points/piece, while the continuous line is characterized by 10 points/piece.

Anyway, the number of available filters inside the generator cannot satisfy every need: often, the smoothing effect can be poor or so significant to adversely affect the power spectrum of the signal. To gain satisfying performance, the user should deploy an external filter specifically designed to match the features of the signal that he intends to play. This approach can, however, be very expensive. On the other hand, the use of hand-made filters manufactured in laboratory through printed-circuit-boards connection of discrete components can lead to poor results.

Alternatively, to enhance the integrity of the noise signal, the piece-constant waveform should be played imposing a generation frequency much greater than the related Nyquist frequency. This approach represents a simple and promising solution that also simplifies the work of the low pass filter in smoothing the abrupt voltage steps. The available filter becomes more effective because its intervention is now addressed to a kind of refinement [20].

In order to increase the generation frequency without modifying the power spectral density of the signal, appropriate interpolation algorithms are deployed. The interpolation should grant uniform gain within the bandwidth in which the noise is assigned, and a fast decaying frequency response in the transition bandwidth. Nonetheless, the computational burden of the interpolation should be thoroughly limited in order to grant real-time processing execution in the generation chain. To this end the discrete noise sequence is first up-sampled, i.e. prolonged by inserting a given number of zeroes between each couple of samples; the number of zeroes inserted between adjacent samples is equal to $F_{U p}-1$, being $F_{U p}$ the upsampling factor. The prolonged sequence is hence convolved with an opportune interpolating window in order to obtain the interpolated version of the noise. The choice of the type and length of the window should satisfy a trade-off between the accuracy of the spectral features of the noise and the cost required to the processing task. The 


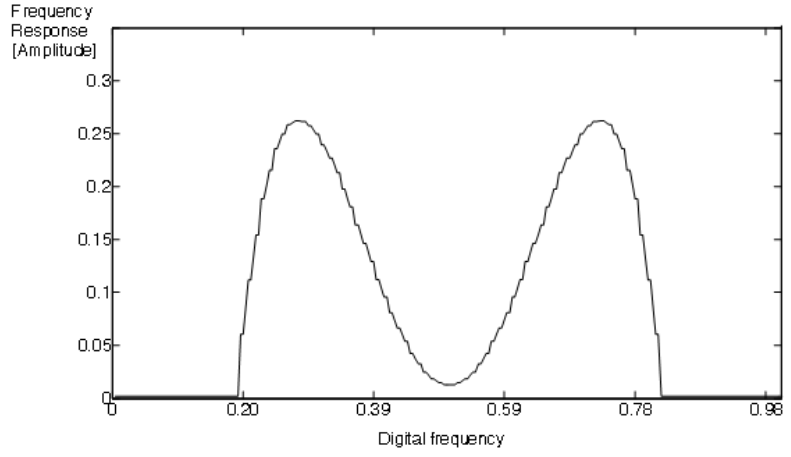

Figure 5. Power spectral density of a noise signal defined in the digital frequency interval $(0.19,0.81)$ by the polynomial $y(x)=100\left(x^{2}-x+0.16\right)\left(x^{2}-\right.$ $x+0.24)+0.1$; in the complementary intervals, i.e. $(0,0.19)$ and $(0.81,1)$, the power spectral density level is 100 times smaller than the peak power density.

time necessary for interpolation depends on the processing capabilities of the processor inside the generator, the length of the interpolation window, and the required generation frequency.

For the sake of clarity a further example of noise is presented in Figure 4, which shows the time domain evolution of both a piece-constant noise (dotted line) and of its version obtained after up-sampling by a factor $F_{U p}=10$ and interpolation with a Chebyshev window (solid line).

The noise is characterized by the imposed PSD shown in Figure 5, that has been defined in terms of a quartic polynomial function in the digital frequency interval $(0.20$, 0.80 ) and a uniform noise floor with level 100 times smaller than the peak power density in $(0,0.20)$ and $(0.80,1)$. In order to highlight the benefits that should be expected when up-sampling and interpolation processing are performed in Figure 6, the simulated power spectral densities curves of both versions of noise are given: the dotted line is the PSD of the piece-constant noise, the solid line is the noise up-sampled by $F_{U p}=10$ and interpolated with a Chebyshev window of length equal to $4 F_{U p}$. The performance of the interpolation window increases with the length of the window; on the other side a longer interpolation window is more demanding in terms of

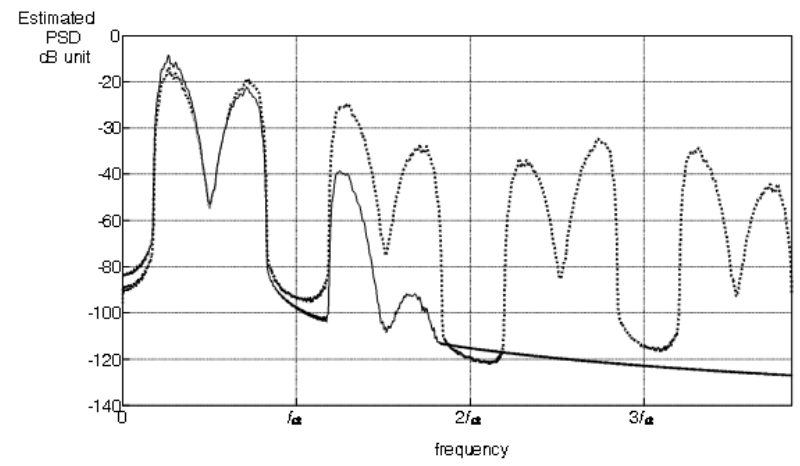

Figure. 6. Portion of the spectrum of the noise signal (dotted line) and of its up-sampled and interpolated version obtained with $F_{U p}=10$, and a Chebychev window of length $4 F_{U p}$ (solid line); in the generation the expected spectrum is replicated at a pace equal to the adopted clock rate $f_{c k}$. This undesired distortion effect is effectively mitigated by up-sampling and interpolation.

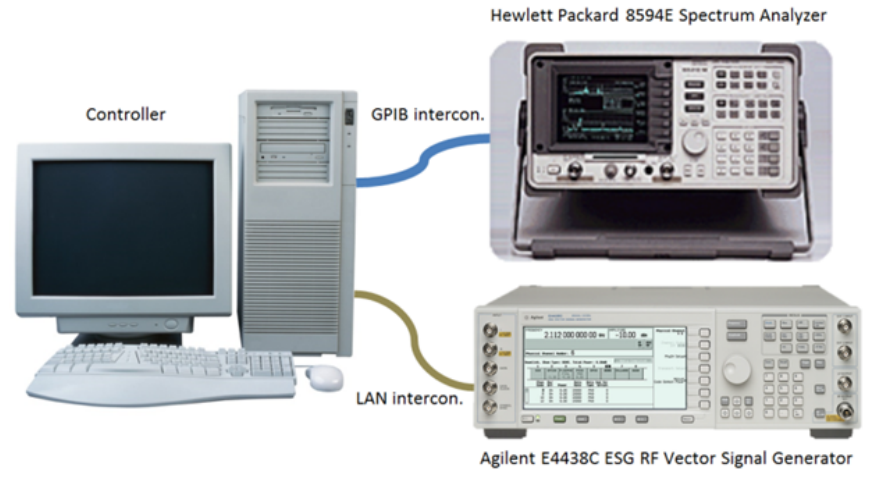

Figure 7. Set-up arranged to carry out the experiments.

computational capabilities and can be not compatible with real-time processing constraints. Note that interpolation has an inherent low-pass filter behaviour, thus it also introduces some attenuation within the bandwidth $B$. The aforementioned attenuation can be tolerated whenever the signal does not involve relevant spectral contributions next to the upper limit of the selected band.

The work of the analogue output filter should further improve the coherence between the expected output power spectrum and the reproducible one.

\section{EXPERIMENTAL SET-UP}

In order to confirm the results of the simulations shown in the previous section an experimental set-up has been arranged and some experiments have been carried out. The experimental set-up, illustrated in Figure 7, has included an $\mathrm{RF}$ vector signal generator (Agilent E4438C ESG), equipped with a 14 bit digital to analogue converter (DAC) capable of functioning with a clock rate up to $100 \mathrm{MHz}$, and a spectrum analyser (Hewlett Packard 8594E).

The vector signal generator can play RF signals that combine I and Q baseband modulating signals, which can be created by means of the internal 14 bit DAC. To be exact the I and Q components of the modulated RF signal can be arbitrary defined by the user. The I and Q data points have to be created in a binary format, that is signed 2 'complement 2-byte integer values in the range $(-32767$, +32767 ), big endian byte order. Then the I and Q data points have to be interleaved in a single array in order to create a data waveform that has to be successively downloaded to the local memory of the generator via ftp using the internal generator web server. In the experimental tests, only the baseband section of the vector generator has been exploited. In particular, the synthesized noise has been assigned to the I component and a zero Q component has been inserted to complete the data waveform. For the sake of clarity, the Matlab ${ }^{\circledR}$ script adopted to prepare the data that are finally downloaded into the memory of the vector generator is given in Appendix A.

The analogue version of the I component played by the internal DAC has been picked-up at the auxiliary I output connector. (It is worth noting, however, that for applications that require noise residing in a radio-frequency band according to given patterns, it is sufficient to 

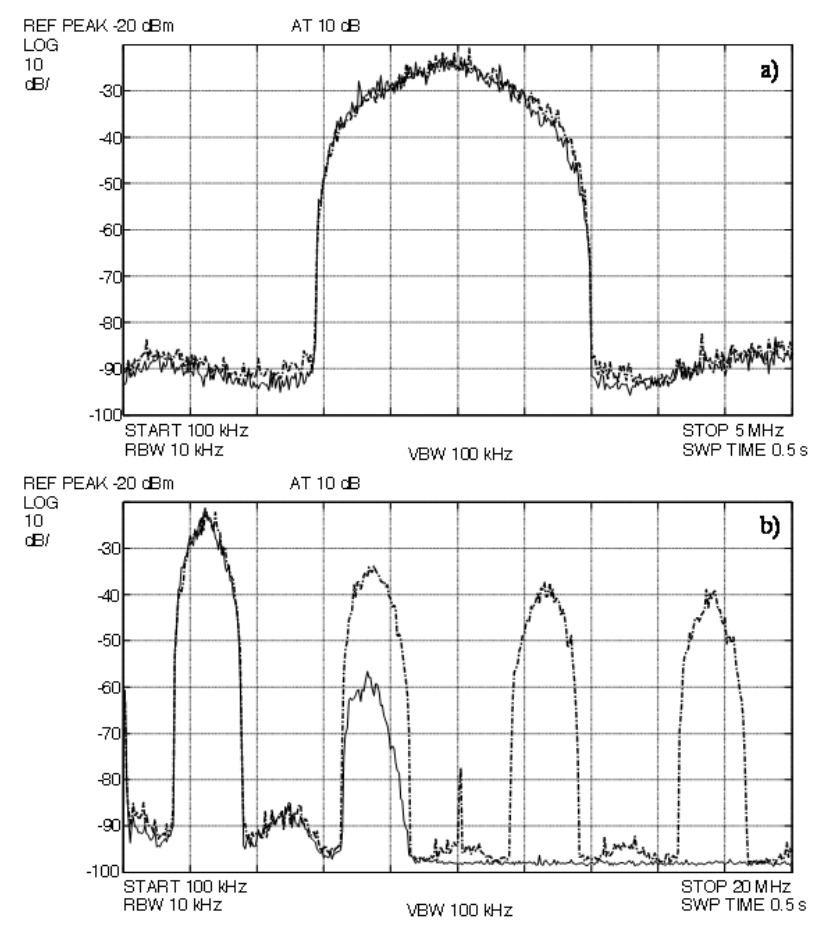

Figure 8. Power spectral density of the noise, characterized by a triangular power spectral density, produced by the DAC internal to the adopted generator. The dashed line is the noise played using a generation frequency equal to $10 \mathrm{MHz}$, the solid line instead is the noise obtained after upsampling by $F_{U p}=10$, interpolation with a Chebyshev window of length equal to $4 F_{U p}$, and played using a generation frequency equal to $100 \mathrm{MHz}$. In a) the analysed frequency interval ranges from $100 \mathrm{kHz}$ up to $5 \mathrm{MHz}$, while in b) from $100 \mathrm{kHz}$ up to $20 \mathrm{MHz}$ in order to highlight the benefits due to the proposed generation strategy in reducing the alias contribution due to the inherent functioning of the DAC. Note the weak spectral line at $10 \mathrm{MHz}$ that characterizes the dashed trace: it witnesses the adoption of a generation frequency equal to $10 \mathrm{MHz}$ for that signal.

synthesize the baseband version of the noise and use a single sideband (SSB) modulation approach to transpose it at RF frequencies).

The first experiments shown hereafter are related to the digital sequence describing the coloured noise characterized by a triangular PSD already discussed in Section 2. For the purposes of the test a very long sequence made up of 5242880 waveform points has been adopted. The noise has been spread in a bandwidth $B=5 \mathrm{MHz}$, and hence played with a generation frequency equal to $10 \mathrm{MHz}$. Successively, also the noise signal obtained after up-sampling by $F_{U p}=10$ and interpolation with a Chebyshev window of length equal to $4 F_{U p}$ has been downloaded and played, this time using a generation frequency equal to $100 \mathrm{MHz}$. No auxiliary analogue filters have been exploited to smooth the effects due to the inherent DAC functioning. Both signals have been picked up at the baseband I output connector and measured by means of the spectrum analyser. Figure 8a shows the power spectrum of the two version of the noise in the frequency range $(100 \mathrm{kHz}, 5 \mathrm{MHz})$; Figure $8 \mathrm{~b}$ shows instead their power spectrum in a wider frequency range, namely $(100 \mathrm{kHz}, 20 \mathrm{MHz})$. The benefits assured by the proposed approach and already highlighted through simulations are proved also by the experimental tests, that confirm the significant reduction of the power spectral
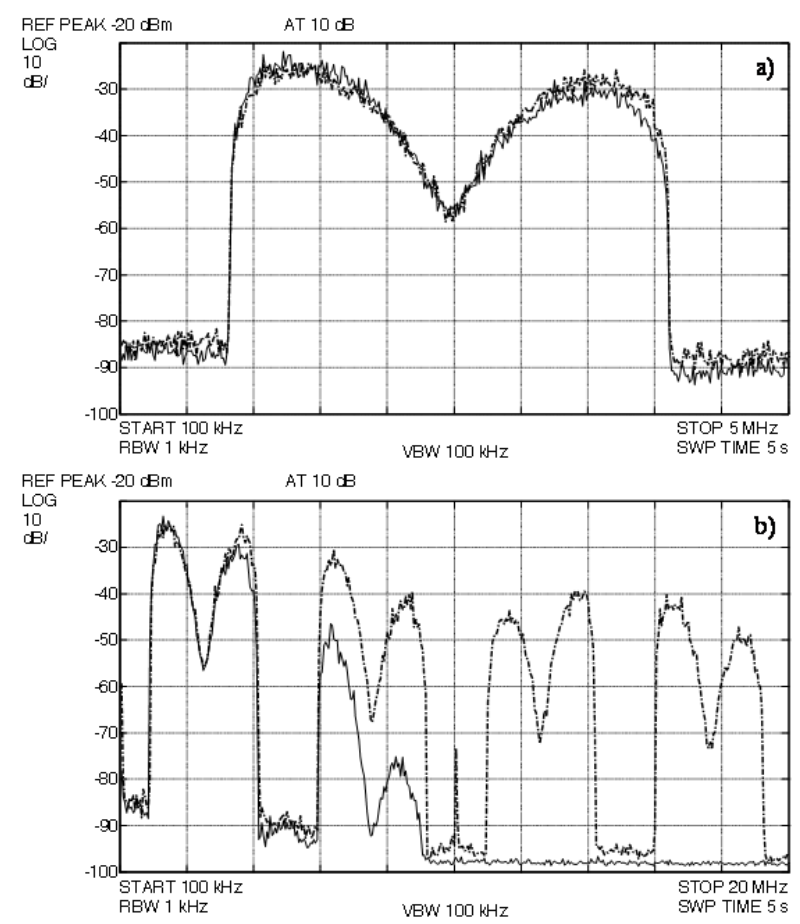

Figure 9. Power spectral density of the noise, characterized by a quartic polynomial power spectral density, produced by the DAC internal to the adopted generator. The dashed line is the noise played using a generation frequency equal to $10 \mathrm{MHz}$, the solid line instead is the noise obtained after upsampling by $F_{U p}=10$, interpolation with a Chebyshev window of length equal to $4 F_{U p}$, and played using a generation frequency equal to $100 \mathrm{MHz}$. In a) the analysed frequency interval ranges from $100 \mathrm{kHz}$ up to $5 \mathrm{MHz}$, while in b) from $100 \mathrm{kHz}$ up to $20 \mathrm{MHz}$ in order to highlight the benefits due to the proposed generation strategy in reducing the alias contribution due to the inherent functioning of the DAC. Once more the weak spectral line at $10 \mathrm{MHz}$ that characterizes the dashed trace witnesses the adoption of a generation frequency equal to $10 \mathrm{MHz}$.

leakage outside the band of interest, obtained without any intervention of external low-pass filters. In addition, further test results are given Figure $9 \mathrm{a}$ and $9 \mathrm{~b}$, which report the power spectrum of the noise characterized by the quartic polynomial power spectral density, already discussed in Section 3.

\section{CONCLUSIONS}

The paper has presented an approach to generate noise with imposed spectral characteristics by means of an arbitrary waveform generator. The proposed approach requires a first stage devoted to the synthesis of the noise in a digital form, in which the output noise is achieved filtering a digital white noise signal. The spectrum of the output noise is shaped by means of a filter suitably designed in order to accomplish the specified spectral features, given in terms of occupied bandwidth and power levels in the selected frequency band.

At the reproduction stage it has been taken into account that (i) the reproduction of any digital signal in analogue form by the DAC internal to arbitrary waveform generators introduces distortion that has to be removed, (ii) for several applications the use of the general purpose filters available on the arbitrary generator leads to poor results, 
(iii) the use of external low-pass filters reveals to be an expensive solution.

Hence, an additional processing stage has been demanded to the processor internal to the generator in order to upsample and interpolate the digital noise before its reproduction. Thanks to this additional processing stage it has been shown that the action of the general purpose lowpass filter internal to the generator is relieved.

It is worth noting that the strength of the proposed approach consists in the distribution between the two stages of the computation needed to suitably synthesize the noise. Actually, the two stages are characterized by different demands in terms of processing rapidity. The first stage is less demanding and allows the consideration of digital filters with adequate length and related heavy computational burden to shape the spectrum of the noise. At the second stage the stream of sample values addressed to the DAC is increased by a factor equal to the up-sampling factor $F_{U p}$ and convolved with an interpolating window; in order to grant real time functioning a minor computational burden is a vital requirement for the processing at this second stage.

The proposed experimental setup has demonstrated the effectiveness of the proposed approach even if it does not work in streaming and is limited in terms of the length of the generated sequence. Future developments will be oriented to the design of a complete coloured noise generation circuit, without limits on the length of the generated sequence, implemented on Field Programmable Gate Arrays, a low cost technology that has been demonstrated ideal for the implementation of fast and efficient circuits, [21]-[23].

\section{APPENDIX A}

Matlab ${ }^{\circledR}$ script adopted to prepare the data that are downloaded into the memory of the vector generator.

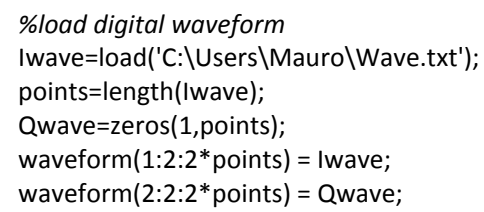

\section{REFERENCES}

[1] Dong-U Lee, Wayne Luk, John D. Villasenor, Peter Y.K. Cheung "A Gaussian noise generator for hardware based simulations," IEEE Transactions on Computers, vol. 53, No. 12, Dec. 2004, pp. 1523-1534.

[2] Dong-U Lee, Wayne Luk, John D. Villasenor, Guanglie Zang, Philip H. W. Leong, "A hardware Gaussian noise generator using the Wallace method," IEEE Transactions on Very Large Scale Integration (VLSI), vol. 13, No. 8, August 2005, pp. 911-920.

[3] C. Imperiale, "Programmable uniform-Gaussian, quasiwhite noise generator and data acquisition-processing system for electrophysiology," Measurement, Vol.20, n.3, March 1997, pp.149-163.

[4] P. Robbins, "Using Noise for RF receiver built-in test applications," Microwave Journal, February 2004.

[5] L. Angrisani, M. D’Apuzzo, M. D’Arco, "Modelling timing jitter effects in digital to analog converters," IEEE Transactions on Instrumentation and Measurement, Vol.58, n.2, February 2009, pp.330-336.

[6] L. De Vito, S. Rapuano, M. Villanacci, "Prototype of an automatic digital modulation classifier embedded in a realtime spectrum analyzer," IEEE Transactions on Instrumentation and Measurement, Vol.59, October 2010, pp.2639-2651.

[7] D.A. Lampasi, L. Podestà, P. Carbone, "Synthesis of binary test sequences with good spectral properties using an evolutionary algorithm," Measurement, Vol.39, n.3, April 2006, pp.245-251.

[8] IEEE Std 1241-2000 "IEEE Standard for terminology and test methods for analog-to-digital converters", IEEE Institute of Electrical and Electronics Engineers Inc., Published 13 June 2001.

[9] A. Baccigalupi, M. D’Arco, A. Liccardo, M. Vadursi, "Testing high resolution DACs: a contribution to draft standard IEEE P1658,” Measurement, Vol.44, n.6, July 2011, pp.1044-1052.

[10] L. Krause, "Effective quantization by averaging and dithering," Measurement, Vol.39, n.8, October 2006, pp.681-694.

[11] P. Carbone, D. Petri "Mean value and variance of noisy quantized data," Measurement, Vol.23, n.3, April 1998, pp.131-144.

[12] R. C. Martins, A. C. Serra, "ADC characterization by using the histogram test stimulated by Gaussian noise: Theory and experimental results," Measurement, Vol.27, n.4, June 2000, pp.291-300.

[13] N. J. Kasdin, "Discrete simulation of colored noise and stochastic processes and $1 / \mathrm{f}^{\alpha}$ power law noise generation," Proceedings of the IEEE, vol. 83, No. 5, May 1995, pp. 802827.

[14] L.F. Pender, H.J. Wintle, "Electrical 1/f noise in insulating polymers," Journal of Applied Physics, 1979, Vol 50, No.1, pp.361-368.

[15] G. Corsini, R. Saletti, “A $1 / \mathrm{f}^{\prime}$ power spectrum noise sequence generator," IEEE Transactions on Instrumentation and Measurement, Vol. 37, No. 4, Dec 1988, pp. 615-619.

[16] D. B. Percival, "Simulating Gaussian random processes with specified spectra," Comput. Sci. Stat., vol. 24, pp. 534-538, 1992.

[17] L. Angrisani, M. D’Arco, E. Napoli, "Programmable power spectral density noise source," 19th IMEKO TC 4 Symposium and 17th IWADC Workshop Advances in 
Instrumentation and Sensors Interoperability July 18-19, 2013, Barcelona, Spain.

[18] M. D'Apuzzo, M. D’Arco, L. Liccardo, M. Vadursi, "Modelling DAC output waveforms," IEEE Transactions on Instrumentation and Measurement, vol.59, n.11, November 2010, pp.2854-2862.

[19] L. Angrisani, M. D’Apuzzo, M. D’Arco, "Fast transformation for DAC parameter identification," IEEE Transactions on Instrumentation and Measurement, Vol.55, December 2006, pp.2007-2013.

[20] Domenico Luca Carnì, Luca De Vito, Sergio Rapuano, “A portable instrument for automatic detection and classification of telecommunication signals," Measurement, Vol.45, No.2, February 2012, pp.182-189.

[21] A. Baccigalupi, M. D’Arco, A. Liccardo, M. Vadursi, “Test equipment for DAC performance assessment: design and characterization," IEEE Transactions on Instrumentation and Measurement, vol.59, n.5, May 2010, pp.1027-1034. M. Fazio, S.L. Rota, Metrology on stamps, Phys. Educ. 30 (1995) pp.289-297.

[22] M. Genovese, E. Napoli, D. De Caro, N. Petra, A.G.M. Strollo, "Analysis and comparison of Direct Digital Frequency Synthesizers implemented on FPGA," Integration, the VLSI Journal, doi:10.1016/j.vlsi.2013.09.001.

[23] M. D'Arco, M. Genovese, E. Napoli, M. Vadursi, "Design and Implementation of a Preprocessing Circuit for Bandpass Signals Acquisition," IEEE Transactions on Instrumentation and Measurement, 2014, Vol 63, No.2, pp.287-294. 